\title{
ASYMPTOTIC BEHAVIOURS OF STOCHASTIC DIFFERENTIAL DELAY EQUATIONS
}

\author{
Yi Shen and Xuerong Mao
}

\begin{abstract}
Most of the existing results on stochastic stability use a single Lyapunov function, but we shall instead use multiple Lyapunov functions in this paper. We shall establish the sufficient condition, in terms of multiple Lyapunov functions, for the asymptotic behaviours of solutions of stochastic differential delay equations. Moreover, from them follow many effective criteria on stochastic asymptotic stability, which enable us to construct the Lyapunov functions much more easily in applications. In particular, the well-known classical theorem on stochastic asymptotic stability is a special case of our more general results. These show clearly the power of our new results. Two examples are also given for illustration.
\end{abstract}

KeyWords: Lyapunov function, asymptotic stability, semi-martingale convergence theorem, Itô formula.

\section{INTRODUCTION}

Since Itô introduced his stochastic calculus more than 50 years ago, the theory of stochastic differential equations has been developed very quickly. In particular, Lyapunov's second method has been developed to deal with stochastic stability by many authors, and we here only mention Arnold [1], Friedman [2], Has'minskii [3], Kolmanovskii and Myshkis [4], Kushner [5], Ladde [6], Lakshmikantham et al. [7], Mao [10-13] and Mohammed [15]. Most of the existing results on stochastic stability use a single Lyapunov function. Recently, Mao [14] discussed the asymptotic stability of stochastic system via multiple Lyapunov functions, but the results obtained in [14] can not be applied to the stochastic differential delay equations. The main aim of this paper is to establish the sufficient condition, in terms of multiple Lyapunov functions, for the asymptotic behaviours of solutions of stochastic differential delay equations. Furthermore, from them follow many useful results on stochastic asymptotic stability, which en-

Manuscript received March 7, 2005; accepted November 12, 2005.

Yi Shen is with Department of Control Science and Engineering, Huazhong University of Science and Technology, Wuhan, Hubei, 430074, China.

Xuerong Mao is with Department of Statistics and Modelling Science, University of Strathclyde, Glasgow G1 1XH, UK (e-mail: xuerong@stams.strath.ac.uk). able us to construct the Lyapunov functions much more easily in applications. In particular, we shall show in Section 4 below that the well-known classical theorem on stochastic asymptotic stability is a special case of our more general results. These show clearly the power of our new results. It should also be pointed out that the idea of using two Lyapunov functions in the study of stability of ordinary differential equations can be found in Salvadori [16] and, more generally, the idea of using vector Lyapunov functions can be found in Lakshmikantham et al. [7].

\section{MAIN RESULTS}

Throughout this paper, unless otherwise specified, we let $\left(\Omega, F,\left\{F_{t}\right\}_{t \geq 0}, P\right)$ be a complete probability space with a filtration $\left\{F_{t}\right\}_{t \geq 0}$ satisfying the usual conditions (i.e., it is increasing and right continuous while $F_{0}$ contains all $P$-null sets). Let $B(t)=\left(B_{1}(t), \cdots, B_{m}(t)\right)^{T}$ be an $m$-dimensional Brownian motion defined on the probability space. Let $|\cdot|$ denote the Euclidean norm in $R^{n}$. If $A$ is a vector or matrix, its transpose is denoted by $A^{T}$. If $A$ is a matrix, its trace norm is denoted by $|A|=\sqrt{\operatorname{trace}\left(A^{T} A\right)}$. Let $\tau>0$ and $C\left([-\tau, 0] ; R^{n}\right)$ denote the family of all continuous $R^{n}$-valued functions on $[-\tau, 0]$. Let $C_{F_{0}}^{b}\left([-\tau, 0] ; R^{n}\right)$ be the family of all $F_{0}$-measurable bounded $C\left([-\tau, 0] ; R^{n}\right)$-valued random variables $\xi=\{\xi(\theta):-\tau \leq \theta \leq 0\}$. 
Consider the following non-autonomous $n$-dimensional stochastic differential delay equation

$$
d x(t)=f(t, x(t), x(t-\tau)) d t+g(t, x(t), x(t-\tau)) d B(t)
$$

on $t \geq 0$ with initial data $\{x(\theta):-\tau \leq \theta \leq 0\}$ $=\xi \in C_{F_{0}}^{b}\left([-\tau, 0] ; R^{n}\right)$, where $f: R_{+} \times R^{n} \times R^{n} \rightarrow R^{n}$ and $g: R_{+} \times R^{n} \times R^{n} \rightarrow R^{n \times m}$.

We assume that $f$ and $g$ satisfy the following conditions:

Hypothesis (H). Both $f$ and $g$ are Borel-measurable functions. They satisfy the local Lipschitz condition and the linear growth condition. That is, for each $k=1,2, \ldots$, there is a $c_{k}>0$ such that

$$
\begin{aligned}
& |f(t, x, y)-f(t, \bar{x}, \bar{y})|+|g(t, x, y)-g(t, \bar{x}, \bar{y})| \\
& \quad \leq c_{k}(|x-\bar{x}|+|y-\bar{y}|)
\end{aligned}
$$

for all $t \geq 0$ and those $x . \quad y, \bar{x}, \bar{y} \in R^{n}$ with $\max \{x, y, \bar{x}, \bar{y}\} \leq k$ and there is moreover a $c>0$ such that

$$
|f(t, x, y)|+|g(t, x, y)| \leq c(1+|x|+|y|)
$$

for all $(t, x, y) \in R_{+} \times R^{n} \times R^{n}$.

Under hypothesis (H), it is known (see e.g. [12]) that, for any initial data $\{x(\theta):-\tau \leq \theta \leq 0\}=\xi \in C_{F_{0}}^{b}([-\tau, 0]$; $R^{n}$ ), Eq. (1) has a unique continuous solution on $t \geq-\tau$, which is denoted by $x(t ; \xi)$ in this paper.

Let $\kappa$ denote the class of continuous (strictly) increasing functions $\mu$ from $R_{+}$to $R_{+}$with $\mu(0)=0$. Let $\kappa_{\infty}$ denote the class of continuous functions $\mu$ in $\kappa$ with $\mu(r) \rightarrow \infty$ as $r$ $\rightarrow \infty$. We also denote by $L^{1}\left(R_{+} ; R_{+}\right)$the family of all functions $\gamma: R_{+} \rightarrow R_{+}$such that $\int_{0}^{\infty} \gamma(t) d t<\infty$ while we denote by $D\left(R_{+} ; R_{+}\right)$the family of all continuous functions $\eta$ : $R_{+} \rightarrow R_{+}$such that $\int_{0}^{\infty} \eta(t) d t=\infty$. Let $C^{2,1}\left(R_{+} \times R^{n} ; R_{+}\right)$ denote the family of all nonnegative functions $V(t, x)$ on $R_{+}$ $\times R^{n}$ which are continuously twice differentiable in $x$ and once differentiable in $t$. For a given $V \in C^{2,1}\left(R_{+} \times R^{n} ; R_{+}\right)$, define an operator $L V: R_{+} \times R^{n} \times R^{n} \rightarrow R$ by

$$
\begin{aligned}
L V(t, x, y) & =V_{t}(t, x)+V_{x}(t, x) f(t, x, y) \\
& +\frac{1}{2} \operatorname{trace}\left[g^{T}(t, x, y) V_{x x}(t, x) g(t, x, y)\right],
\end{aligned}
$$

where

$$
\begin{aligned}
& V_{t}(t, x)=\frac{\partial V(t, x)}{\partial t} \\
& V_{x}(t, x)=\left(\frac{\partial V(t, x)}{\partial x_{1}}, \ldots, \frac{\partial V(t, x)}{\partial x_{n}}\right), \\
& V_{x x}(t, x)=\left(\frac{\partial^{2} V(t, x)}{\partial x_{i} \partial x_{j}}\right)_{n \times n} .
\end{aligned}
$$

It should be emphasised that the operator $L V$ is defined on $R_{+} \times R^{n} \times R^{n}$, although the function $V$ is defined only on $R_{+}$ $\times R^{n}$. In particular, $L V$ is a single operator but not thought as $L$ acting on $V$.

To prove our main result, which is a stochastic version of the well-known LaSalle theorem (see $[8,13]$ ) for locating limit sets of Eq. (1), we will need the following nonnegative semi-martingale convergence theorem established by Liptser and Shiryayer [9].

Lemma 2.1. Let $A(t)$ and $U(t)$ be two continuous adapted increasing processes on $t \geq 0$ with $A(0)=U(0)=0$ a.s. Let $M(t)$ be a real-valued continuous local martingale with $M(0)$ $=0$ a.s. Let $\xi$ be a nonnegative $F_{0}$-measurable random variable such that $E \xi<\infty$. Define

$$
X(t)=\xi+A(t)-U(t)+M(t) \quad \text { for } t \geq 0 .
$$

If $X(t)$ is nonnegative, then

$$
\left\{\lim _{t \rightarrow \infty} A(t)<\infty\right\} \subset\left\{\lim _{t \rightarrow \infty} X(t)<\infty\right\} \cap\left\{\lim _{t \rightarrow \infty} U(t)<\infty\right\} \quad \text { a.s. }
$$

where $B \subset D$ a.s. means $P\left(B \cap D^{c}\right)=0$. In particular, if $\lim _{t \rightarrow \infty} A(t)<\infty$ a.s., then for almost all $\omega \in \Omega$

$$
\lim _{t \rightarrow \infty} X(t, \omega)<\infty, \quad \lim _{t \rightarrow \infty} U(t, \omega)<\infty,
$$

and

$$
\lim _{t \rightarrow \infty} M(t, \omega)<\infty .
$$

Theorem 2.2. Let $(\mathbf{H})$ hold. Assume that there are functions $U, V \in C^{2,1}\left(R_{+} \times R^{n} ; R_{+}\right), \gamma_{1}, \gamma_{2} \in L^{1}\left(R_{+} ; R_{+}\right), w_{1}, w_{2} \in$ $C\left(R_{+} \times R^{\mathrm{n}} ; R_{+}\right), \eta \in D\left(R_{+} ; R_{+}\right)$and $\rho \in C\left(R_{+} ; R_{+}\right)$such that

$$
\begin{aligned}
& L U(t, x, y) \leq \gamma_{1}(t), \\
& L V(t, x, y) \leq \gamma_{2}(t)-w_{1}(t, x)+w_{2}(t, y), \\
& w_{1}(t, x) \geq w_{2}(t+\tau, x),
\end{aligned}
$$

and

$$
\begin{aligned}
\gamma_{2}(t) & -L V(t, x, y)-w_{1}(t, x)+w_{2}(t, y) \\
& +\left|V_{x}(t, x) g(t, x, y)\right|^{2} \geq \eta(t) \rho(U(t, x))
\end{aligned}
$$

for all $(t, x, y) \in R_{+} \times R^{n} \times R^{n}$. Then $\operatorname{ker}(\rho):=\{u \geq 0: \rho(u)=$ $0\} \neq \varnothing$ (as usual $\varnothing$ denotes the empty set), and the solution $x(t ; \xi)$ of Eq. (1) satisfies

$$
\lim _{t \rightarrow \infty} U(t, x(t ; \xi)) \in \operatorname{ker}(\rho) \quad \text { a.s. }
$$

for every $\xi \in C_{F_{0}}^{b}\left([-\tau, 0] ; R^{n}\right)$.

Proof. Fix any initial data $\xi$ and write $x(t ; \xi)=x(t)$ for simplicity. We shall first prove that

$$
\lim _{t \rightarrow \infty} U(t, x(t))<\infty \quad \text { a.s. }
$$


By Itô's formula,

$$
\begin{aligned}
U(t, x(t))= & U(0, \xi)+\int_{0}^{t} L U(s, x(s), x(s-\tau)) d s \\
& +\int_{0}^{t} U_{x}(s, x(s)) g(s, x(s), x(s-\tau)) d B(s) \\
= & U(0, \xi) \\
& +\int_{0}^{t}\left(\gamma_{1}(s) d s-L U(s, x(s), x(s-\tau))\right) d s \\
& +\int_{0}^{t} U_{x}(s, x(s)) g(s, x(s), x(s-\tau)) d B(s)
\end{aligned}
$$

By Lemma 2.1, we know that (7) holds.

Secondly, the following inequality (9) will be proved:

$$
\begin{aligned}
\int_{0}^{\infty} & {\left[\gamma_{2}(s)-L V(s, x(s), x(s-\tau))\right.} \\
& -w_{1}(s, x(s))+w_{2}(s, x(s-\tau)) \\
& \left.+\left|V_{x}(s, x(s)) g(s, x(s), x(s-\tau))\right|^{2}\right] d s<\infty \text { a.s. (9) }
\end{aligned}
$$

By Itô's formula again, we have

$$
\begin{gathered}
V(t, x(t))=V(0, \xi)+\int_{0}^{t} L V(s, x(s), x(s-\tau)) d s \\
\quad+\int_{0}^{t} V_{x}(s, x(s)) g(s, x(s), x(s-\tau)) d B(s) .
\end{gathered}
$$

Further,

$$
\begin{aligned}
\int_{t-\tau}^{t} w_{2} & (s+\tau, x(s)) d s=\int_{-\tau}^{0} w_{2}(s+\tau, \xi(s)) d s \\
& +\int_{0}^{t}\left[w_{2}(s+\tau, x(s))-w_{2}(s, x(s-\tau))\right] d s .
\end{aligned}
$$

By (10) and (11), we get

$$
\begin{aligned}
V( & , x(t))+\int_{t-\tau}^{t} w_{2}(s+\tau, x(s)) d s \\
= & V(0, \xi)+\int_{-\tau}^{0} w_{2}(s+\tau, \xi(s)) d s \\
& +\int_{0}^{t}\left[w_{2}(s+\tau, x(s))-w_{2}(s, x(s-\tau))\right] d s \\
& +\int_{0}^{t} L V(s, x(s), x(s-\tau)) d s \\
& +\int_{0}^{t} V_{x}(s, x(s)) g(s, x(s), x(s-\tau)) d B(s) \\
\leq & V(0, \xi)+\int_{-\tau}^{0} w_{2}(s+\tau, \xi(s)) d s \\
& +\int_{0}^{t}\left[w_{1}(s, x(s))-w_{2}(s, x(s-\tau))\right] d s \\
& +\int_{0}^{t} L V(s, x(s), x(s-\tau)) d s
\end{aligned}
$$

$$
\begin{gathered}
+\int_{0}^{t} V_{x}(s, x(s)) g(s, x(s), x(s-\tau)) d B(s) \\
=V(0, \xi)+\int_{-\tau}^{0} w_{2}(s+\tau, \xi(s)) d s+\int_{0}^{t} \gamma_{2}(s) d s \\
-\int_{0}^{t}\left[\gamma_{2}(s)-L V(s, x(s), x(s-\tau))\right. \\
\left.\quad-w_{1}(s, x(s))+w_{2}(s, x(s-\tau))\right] d s \\
+\int_{0}^{t} V_{x}(s, x(s)) g(s, x(s), x(s-\tau)) d B(s),
\end{gathered}
$$

where we have used condition (4). By condition (3), applying the nonnegative semi-martingale convergence theorem (Lemma 2.1) to (12) yields

$$
\begin{aligned}
\int_{0}^{\infty} & {\left[\gamma_{2}(s)-L V(s, x(s), x(s-\tau))\right.} \\
& \left.-w_{1}(s, x(s))+w_{2}(s, x(s-\tau))\right] d s<\infty \quad \text { a.s. } \\
-\infty & <\lim _{t \rightarrow \infty} M(t)<\infty \quad \text { a.s. }
\end{aligned}
$$

where $M(t)=\int_{0}^{t} V_{x}(s, x(s)) g(s, x(s), x(s-\tau)) d B(s)$.

For any integer $i \geq 1$, define a stopping time

$$
\tau_{i}=\inf \{t \geq 0:|M(t)| \geq i\}
$$

where throughout this paper we set inf $\varnothing=\infty$. It is clear that $\tau_{i}$ is increasing. By (14), there exists a subset $\Omega_{1}$ of $\Omega$ with $P\left(\Omega_{1}\right)=1$ such that for every $\omega \in \Omega_{1}$ there is an $i(\omega)$ such that

$$
\tau_{i}(\omega)=\infty, \quad \forall i \geq i(\omega) .
$$

On the other hand, for any $t>0$ and $i \geq 1$, we have

$$
\begin{aligned}
& E \int_{0}^{t \wedge \tau_{i}}\left|V_{x}(s, x(s)) g(s, x(s), x(s-\tau))\right|^{2} d s \\
& \quad=E\left|M\left(t \wedge \tau_{i}\right)\right|^{2} \leq i^{2} .
\end{aligned}
$$

Letting $t \rightarrow \infty$ yields

$$
E \int_{0}^{\tau_{i}}\left|V_{x}(s, x(s)) g(s, x(s), x(s-\tau))\right|^{2} d s \leq i^{2},
$$

which implies that

$$
\int_{0}^{\tau_{i}}\left|V_{x}(s, x(s)) g(s, x(s), x(s-\tau))\right|^{2} d s<\infty
$$

holds with probability 1 . Therefore, there is another subset $\Omega_{2}$ of $\Omega$ with $P\left(\Omega_{2}\right)=1$ such that if $\omega \in \Omega_{2}$, (16) holds for any $i \geq 1$. Hence for any $\omega \in \Omega_{1} \cap \Omega_{2}$, we have

$$
\begin{aligned}
& \int_{0}^{\infty}\left|V_{x}(s, x(s, \omega)) g(s, x(s, \omega), x(s-\tau, \omega))\right|^{2} d s \\
& =\int_{0}^{\tau_{i(\omega)}(\omega)}\left|V_{x}(s, x(s, \omega)) g(s, x(s, \omega), x(s-\tau, \omega))\right|^{2} d s \\
& \quad<\infty .
\end{aligned}
$$


Since $P\left(\Omega_{1} \cap \Omega_{2}\right)=1$, we must have

$$
\int_{0}^{\infty}\left|V_{x}(s, x(s)) g(s, x(s), x(s-\tau))\right|^{2} d s<\infty \text { a.s. (17) }
$$

This, together with (13), gives the required result (9).

Thirdly, we shall prove $\operatorname{ker}(\rho) \neq \varnothing$ and (6). It follows from (5) and (9) that

$$
\int_{0}^{\infty} \eta(t) \rho(U(t, x(t))) d t<\infty \quad \text { a.s. }
$$

Since $\rho \in C\left(R_{+} ; R_{+}\right)$, it follows from (7) that

$$
0 \leq \lim _{t \rightarrow \infty} \rho(U(t, x(t)))=\rho\left(\lim _{t \rightarrow \infty} U(t, x(t))\right)<\infty \quad \text { a.s. }
$$

We now claim that

$$
\lim _{t \rightarrow \infty} \rho(U(t, x(t)))=\rho\left(\lim _{t \rightarrow \infty} U(t, x(t))\right)=0 \quad \text { a.s. }
$$

If this is false, there is some $\Omega_{0} \subset \Omega$ with $P\left(\Omega_{0}\right)>0$ such that for any $\omega \in \Omega_{0}$,

$$
\lim _{t \rightarrow \infty} \rho(U(t, x(t, \omega)))>0 .
$$

Therefore, for any $\omega \in \Omega_{0}$, there exist $\varepsilon(\omega)>0$ and $T(\omega)>$ 0 such that for $t \geq T(\omega)$ we have

$$
\rho(U(t, x(t, \omega)))>\varepsilon(\omega) .
$$

So

$$
\int_{0}^{\infty} \eta(t) \rho(U(t, x(t, \omega))) d t \geq \varepsilon(\omega) \int_{T(\omega)}^{\infty} \eta(t) d t=\infty .
$$

which contradicts (18). Hence (19) must hold. It now follows from (19) immediately that $\operatorname{ker}(\rho) \neq \varnothing$ and

$$
\lim _{t \rightarrow \infty} U(t, x(t)) \in \operatorname{ker}(\rho) \text { a.s. }
$$

as required. This completes the proof.

Remark 1. Theorem 2.2 can be regarded as the stochastic version of the LaSalle theorem and the extension of the main results in $[8,13,14]$. Apparently, when the time delay $\tau=0$, Eq. (1) reduces to a delay-free equation which was discussed in [14]. In this case, the results in the paper reduce to the corresponding results in [14].

\section{ASYMPTOTIC STABILITY}

In this section we shall show that Theorem 2.2 can be applied to study asymptotic stability. For the stability purpose, we impose a standing hypothesis:

$$
f(t, 0,0)=0 \quad \text { and } \quad g(t, 0,0)=0 \quad \forall t \geq 0 .
$$

Hence Eq. (1) admits a trivial solution $x(t)=0$. In this paper when we say that (the trivial solution of) Eq. (1) is asymptotically stable with probability 1 we mean that for any initial data $\xi \in C_{F_{0}}^{b}\left([-\tau, 0] ; R^{n}\right)$, the solution of Eq. (1) obeys

$$
\lim _{t \rightarrow \infty}|x(t ; \xi)|=0 \quad \text { a.s. }
$$

Theorem 3.1. Let $(\mathbf{H})$ hold and assume that all the conditions of Theorem 2.2 hold. If

$$
\operatorname{ker}(\rho)=\{0\},
$$

and, moreover, there is a function $\mu \in \kappa$ such that for any $(t, x) \in R_{+} \times R^{n}$,

$$
\mu(|x|) \leq U(t, x),
$$

then for any $\xi \in C_{F_{0}}^{b}\left([-\tau, 0] ; R^{n}\right)$,

$$
\lim _{t \rightarrow \infty}|x(t ; \xi)|=0 \quad \text { a.s. }
$$

The proof is straightforward and hence omitted.

Theorem 3.2. Let $\mathbf{( H )}$ hold. Assume that there are functions $U, V \in C^{2,1}\left(R_{+} \times R^{n} ; R_{+}\right), \gamma_{1}, \gamma_{2} \in L^{1}\left(R_{+} ; R_{+}\right), w_{1}, w_{2} \in$ $C\left(R_{+} \times R^{n} ; R_{+}\right), \eta \in D\left(R_{+} ; R_{+}\right)$and $\mu_{1}, \mu_{2}, \mu_{3} \in \kappa$ such that for all $(t, x, y) \in R_{+} \times R^{n} \times R^{n}$, (2)-(4) hold and, moreover,

$$
\mu_{1}(|x|) \leq U(t, x) \leq \mu_{2}(|x|),
$$

and

$$
\begin{aligned}
\gamma_{2}(t) & -L V(t, x, y)-w_{1}(t, x)+w_{2}(t, y) \\
& +\left|V_{x}(t, x) g(t, x, y)\right|^{2} \geq \eta(t) \mu_{3}(|x|) .
\end{aligned}
$$

Then, for any $\xi \in C_{F_{0}}^{b}\left([-\tau, 0] ; R^{n}\right)$, the solution $x(t ; \xi)$ of Eq. (1) satisfies

$$
\lim _{t \rightarrow \infty}|x(t ; \xi)|=0 \quad \text { a.s. }
$$

Proof. Without loss of generality we may assume that $\mu_{2} \in$ $\kappa_{\infty}$ (otherwise we can replace $\mu_{2}$ by $\mu_{2}+\mu$ which is in $\kappa_{\infty}$, where $\mu(r)=r$ for $r \geq 0)$. Therefore, its inverse function $\mu_{2}^{-1} \in \kappa_{\infty}$. It follows from (20) that

$$
\mu_{2}^{-1}(U(t, x)) \leq|x| \text {. }
$$

Substituting this into (21) yields

$$
\begin{aligned}
& \gamma_{2}(t)-L V(t, x, y)-w_{1}(t, x)+w_{2}(t, y) \\
& \quad+\left|V_{x}(t, x) g(t, x, y)\right|^{2} \geq \eta(t) \mu_{3}\left(\mu_{2}^{-1}(U(t, x(t)))\right) .
\end{aligned}
$$

Set $\rho(u)=\mu_{3}\left(\mu_{2}^{-1}(u)\right)$ for $u \in R_{+}$, which is in $\kappa$ whence $\operatorname{ker}(\rho)=0$. Hence the required assertion (22) now follows from Theorem 3.1. This completes the proof.

Remark 2. Letting $U=V, w_{1}=w_{2}=0, \gamma_{1}=\gamma_{2}$ in Theorem 
3.2 , we observe that if condition (20) holds, then the asymptotic stability with probability one of Eq. (1) can be guaranteed by

$$
\begin{aligned}
L V(t, x, y) & \leq \gamma_{1}(t) \wedge\left(\gamma_{1}(t)+\left|V_{x}(t, x) g(t, x, y)\right|^{2}\right. \\
& \left.-\eta(t) \mu_{3}(|x|)\right) .
\end{aligned}
$$

In the classical stability theory of stochastic differential equations (see $[1,3,5]$ ), $L V$ is required to be negative definite. But our Theorem 3.2 does not require this negative definite property since $L V$ may take positive values somewhere. Moreover, the term $\left|V_{x}(t, x) g(t, x, y)\right|^{2}$ is connected with the diffusion coefficient $g(t, x, y)$. Therefore, our results reveal the important role of noise in stabilizing the system which is described by (1). Stabilization by noise is a very interesting issue but we will not develop it further in this paper due to page limit and we only refer the reader to Mao [12]. Let us now give an example to illustrate the above features explicitly.

Example 3.3. Consider a 2-dimensional stochastic differential delay equation

$$
\begin{aligned}
d\left(\begin{array}{l}
x_{1}(t) \\
x_{2}(t)
\end{array}\right)= & \left(\begin{array}{c}
-x_{1}(t)+x_{1}(t-\tau) x_{2}(t)+e^{-t} \sin \left(x_{2}(t-\tau)\right) \\
-x_{1}(t-\tau) x_{1}(t)-2 x_{2}(t)
\end{array}\right) d t \\
& +\left(\begin{array}{c}
e^{-t} \sin \left(x_{1}(t-\tau)\right) d B_{1}(t) \\
2 x_{2}(t) d B_{2}(t)
\end{array}\right),
\end{aligned}
$$

where $\left(B_{1}(t), B_{2}(t)\right)$ is a two-dimensional Brownian motion. Let $V(t, x, y)=U(t, x, y)=|x|^{2}$ so (20) is satisfied clearly. It is easy to show that the operator $L V: R_{+} \times R^{2} \times R^{2} \rightarrow R$ has the form

$$
L V(t, x, y)=-2 x_{1}^{2}+2 e^{-t} x_{1} \sin \left(y_{2}\right)+e^{-2 t} \sin \left(y_{1}\right),
$$

which is not negative definite, and in fact $L V(t, x, y)>0$ for some $(t, x, y)$. To apply our result, we note that

$$
L V(t, x, y) \leq-x_{1}^{2}+2 e^{-2 t} \leq 2 e^{-2 t} .
$$

Moreover, compute

$$
\left|V_{x}(t, x) g(t, x, y)\right|^{2}=4 x_{1}^{2} e^{-2 t} \sin ^{2}\left(y_{1}\right)+16 x_{2}^{4} .
$$

So

$$
\begin{aligned}
& \gamma_{1}(t)-L V(t, x, y)+\left|V_{x}(t, x) g(t, x, y)\right|^{2} \\
& \geq x_{1}^{2}+16 x_{2}^{4} \geq \eta(t) \mu_{3}(|x|),
\end{aligned}
$$

where

$$
\begin{aligned}
& \gamma_{1}(t)=2 e^{-2 t}, \quad \eta(t)=1, \\
& \mu_{3}(r)=\min _{|x| \geq r}\left[x_{1}^{2}+16 x_{2}^{4}\right] \quad \text { for } r \geq 0 .
\end{aligned}
$$

In other words, (23) is satisfied. By Theorem 3.2 we can therefore conclude that the solution of Eq. (24) starting from anywhere in $R^{2}$ will tend to zero asymptotically with probability one.

\section{USEFUL COROLLARIES}

When the time delay $\tau=0$, Eq. (1) becomes a stochastic differential equation (without delay)

$$
d x(t)=\bar{f}(t, x(t)) d t+\bar{g}(t, x(t)) d B(t)
$$

on $t \geq 0$ with initial value $x(0)=\xi \in R^{n}$, where

$$
\bar{f}(t, x)=f(t, x, x), \quad \bar{g}(t, x)=g(t, x, x) .
$$

For a given $\bar{V} \in C^{2,1}\left(R \times R^{n} ; R\right)$, define an operator $L \bar{V}: R_{+} \times R^{n} \rightarrow R$ by

$$
\begin{aligned}
L \bar{V}(t, x)= & \bar{V}_{t}(t, x)+\bar{V}_{x}(t, x) \bar{f}(t, x) \\
& +\frac{1}{2} \operatorname{trace}\left[\bar{g}^{T}(t, x) \bar{V}_{x x}(t, x) \bar{g}(t, x)\right] .
\end{aligned}
$$

The following Corollaries 4.1, 4.2, and 4.3 on Eq. (25) follow easily from Theorems 2.2, 3.1, and 3.2, respectively, by setting $w_{1}=w_{2}=0$.

Corollary 4.1. Assume that there are functions $\bar{U}, \bar{V} \in C^{2,1}\left(R_{+} \times R^{n} ; R_{+}\right), \gamma_{1}, \gamma_{2} \in L^{1}\left(R_{+} ; R_{+}\right), \eta \in D\left(R_{+} ;\right.$ $\left.R_{+}\right), \rho \in C\left(R_{+} ; R_{+}\right)$such that, for all $(t, x) \in R_{+} \times R^{n}$,

$$
\begin{aligned}
& L \bar{U}(t, x) \leq \gamma_{1}(t), \\
& L \bar{V}(t, x) \leq \gamma_{2}(t),
\end{aligned}
$$

and

$$
\gamma_{2}(t)-L \bar{V}(t, x)+\left|\bar{V}_{x}(t, x) \bar{g}(t, x)\right|^{2} \geq \eta(t) \rho(\bar{U}(t, x)) .
$$

Then $\operatorname{ker}(\rho)=\{u \geq 0: \rho(u)=0\} \neq \varnothing$, and the solution $x(t ; \xi)$ of Eq. (25) satisfies

$$
\lim _{t \rightarrow \infty} \bar{U}(t, x(t ; \xi)) \in \operatorname{ker}(\rho) \quad \text { a.s. }
$$

for every $\xi \in R^{n}$.

Corollary 4.2. Assume that the conditions of Corollary 4.1 hold. If $\operatorname{ker}(\rho)=0$, and there is moreover a function $\mu \in \kappa$ such that for any $(t, x) \in R_{+} \times R^{n}$

$$
\mu(|x|) \leq \bar{U}(t, x) .
$$

Then, for every $\xi \in R^{n}$, the solution $x(t ; \xi)$ of (25) satisfies

$$
\lim _{t \rightarrow \infty}|x(t ; \xi)|=0 \quad \text { a.s. }
$$


Corollary 4.3. Assume that there are functions $\bar{U}, \bar{V} \in C^{2,1}\left(R_{+} \times R^{n} ; R_{+}\right), \gamma_{1}, \gamma_{2} \in L^{1}\left(R_{+} ; R_{+}\right), \eta \in D\left(R_{+} ;\right.$ $\left.R_{+}\right), \mu_{1}, \mu_{2}, \mu_{3} \in \kappa$ such that, for all $(t, x) \in R_{+} \times R^{n},(26)$ and (27) hold and, moreover,

$$
\mu_{1}(|x|) \leq \bar{U}(t, x) \leq \mu_{2}(|x|),
$$

and

$$
\gamma_{2}(t)-L \bar{V}(t, x)+\left|\bar{V}_{x}(t, x) \bar{g}(t, x)\right|^{2} \geq \eta(t) \mu_{3}(|x|) .
$$

Then, for every $\xi \in R^{n}$, the solution $x(t ; \xi)$ of (25) satisfies

$$
\lim _{t \rightarrow \infty}|x(t ; \xi)|=0 \quad \text { a.s. }
$$

The following corollary using a single Lyapunov function follows from Corollary 4.3 by setting $\bar{V}=\bar{U}$.

Corollary 4.4. Assume that there are functions $\bar{V} \in C^{2,1}\left(R_{+} \times R^{n} ; R_{+}\right), \gamma \in L^{1}\left(R_{+} ; R_{+}\right), \mu_{1}, \mu_{2}, \mu_{3} \in \kappa$ such that for all $(t, x) \in R_{+} \times R^{n}$,

$$
\mu_{1}(|x|) \leq \bar{V}(t, x) \leq \mu_{2}(|x|),
$$

and

$$
L \bar{V}(t, x) \leq \gamma(t) \wedge\left[\gamma(t)+\left|\bar{V}_{x}(t, x) \bar{g}(t, x)\right|^{2}-\mu_{3}(|x|)\right] .
$$

Then, for every $\xi \in R^{n}$, the solution $x(t ; \xi)$ of (25) satisfies

$$
\lim _{t \rightarrow \infty}|x(t ; \xi)|=0 \quad \text { a.s. }
$$

To see the important contributions of this paper, let us recall the well-known classical result on the stochastically asymptotic stability (see e.g. Arnold [1], Has'minskii [3], or Kushner [5]).

Theorem 4.5. Assume that there are functions $\bar{V} \in C^{2,1}\left(R_{+} \times R^{n} ; R_{+}\right), \mu_{1}, \mu_{2} \in \kappa_{\infty}, \mu_{3} \in \kappa$ such that, for all $(t, x) \in R_{+} \times R^{n},(30)$ holds and

$$
L \bar{V}(t, x) \leq-\mu_{3}(|x|) \text {. }
$$

Then, for every $\xi \in R^{n}$, the solution $x(t ; \xi)$ of (26) satisfies

$$
\lim _{t \rightarrow \infty}|x(t ; \xi)|=0 \quad \text { a.s. }
$$

Remark 3. It is clear that Corollary 4.4 is an improvement of this classical Theorem (see Remark 2 as well).

\section{AN ILLUSTRATIVE EXAMPLE}

In this section, let us discuss an example to illustrate that our results can also be applied to deal with partial stability.

Example 5.1. Consider a two dimensional stochastic dif- ferential delay equation

$$
\left\{\begin{array}{l}
d x_{1}(t)=-2 x_{1}(t) \sin ^{2} t d t+\exp (-t) \sin x_{2}(t-\tau) d B_{1}(t), \\
d x_{2}(t)=\left(-x_{2}(t)+x_{2}(t-\tau)\right) d t+\sqrt{2} x_{1}(t) \sin t d B_{2}(t),
\end{array}\right.
$$

where $\left(B_{1}, B_{2}\right)$ is a two-dimensional Brownian motion.

Construct the functions $V(t, x)=x_{1}^{2}+x_{2}^{2}$ and $U(t, x)=x_{1}^{2}$, where $x=\left(x_{1}, x_{2}\right)^{T}$ and the following $y=\left(y_{1}\right.$, $\left.y_{2}\right)^{T}$. Compute

$$
\begin{aligned}
L V(t, x, y)= & 2 x_{1}\left(-2 x_{1} \sin ^{2} t\right)+2 x_{2}\left(-x_{2}+y_{2}\right) \\
& +\exp (-2 t) \sin ^{2} y_{2}+2 x_{1}^{2} \sin ^{2} t \\
= & \exp (-2 t) \sin ^{2} y_{2}-2 x_{2}^{2}+2 x_{2} y_{2}-2 x_{1}^{2} \sin ^{2} t \\
\leq & \exp (-2 t)-x_{2}^{2}+y_{2}^{2}-2 x_{1}^{2} \sin ^{2} t \\
\leq & \exp (-2 t)-x_{2}^{2}+y_{2}^{2}
\end{aligned}
$$

and

$$
L U(t, x, y)=2 x_{1}\left(-2 x_{1} \sin ^{2} t\right)+\exp (-2 t) \sin ^{2} y_{2}
$$$$
\leq \exp (-2 t)-4 x_{1}^{2} \sin ^{2} t \leq \exp (-2 t) \text {. }
$$

Let $\quad \gamma_{1}(t)=\gamma_{2}(t)=\exp (-2 t), w_{1}(t, x)=x_{2}^{2}, w_{2}(t, y)=y_{2}^{2}$. We have

$$
\begin{aligned}
\gamma_{2}(t) & -L V(t, x, y)-w_{1}(t, x)+w_{2}(t, y) \\
& +\left|V_{x}(t, x) g(t, x, y)\right|^{2} \geq 2 x_{1}^{2} \sin ^{2} t \\
= & 2 \sin ^{2} t U(t, x) .
\end{aligned}
$$

Set $\eta(t)=2 \sin ^{2} t$. The conditions of Theorem 2.2 are all satisfied. So the solution $x(t ; \xi)$ of Eq. (38) has the property that

$$
\lim _{t \rightarrow \infty}\left|x_{1}(t ; \xi)\right|=0 \quad \text { a.s. }
$$

for every $\xi \in C_{F_{0}}^{b}\left([-\tau, 0] ; R^{2}\right)$.

\section{ACKNOWLEDGMENTS}

The authors would like to thank the referees and the editors for their helpful suggestions. The authors also wish to thank the financial supports from the National Natural Science Foundation of China (No. 60074008, 60274007) as well as the EPSRC (UK) and the Royal Society.

\section{REFERENCES}

1. Arnold, L., Stochastic Differential Equations: Theory 
and Applications, John Wiley and Sons, NY, U.S.A. (1972).

2. Friedman, A., Stochastic Differential Equations and Their Applications, Vol. 2, Academic Press, NY, U.S.A. (1976).

3. Has'minskii, R.Z., Stochastic Stability of Differential Equations, Sijthoff and Nordhoff (1981).

4. Kolmanovskii, V. and A. Myshkis, Introducion to the Theory and Applications of Functional Differential Equations, Kluwer Academic Publishers, London, U.K. (1999).

5. Kushner, H.J., Stochastic Stability and Control, Academic Press, NY, U.S.A. (1967).

6. Ladde, G.S. and V. Lakshmikantham, Random Differential Inequalities, Academic Press, NY, U.S.A. (1980).

7. Lakshmikantham, V., V.M. Martrosov, and A. Sivasundaram, Vector Lyapunov Functions and Stability Analysis of Nonlinear Systems, Kluwer Academic Publishers, Dordrecht (1991).

8. Lasalle, J.P., "Stability Theory of Ordinary Differential Equations," J. Diff. Equat.s, Vol. 4, pp. 57-65 (1968).

9. Liptser, R.Sh. and A.N. Shiryayew, Theory of Martingales, Kluwer Academic Publishers, London, U.K. (1989).

10. Mao, X., Stability of Stochastic Differential Equations with Respect to Semi-Martingales, Longman Scientific and Technical (1991).

11. Mao, X., Exponential Stability of Stochastic Differential Equations, Marcel Dekker (1994).

12. Mao, X., Stochastic Differential Equations and Applications, Horwood (1997).

13. Mao, X., "Stochastic Versions of the LaSalle Theorem," J. Diff. Equat., Vol. 153, pp. 175- 195 (1999).

14. Mao, X., "Some Contributions to Stochastic Asymptotic Stability and Boundedness via Multiple Lyapunov Functions," J. Math. Anal. Appl., Vol. 260, pp. 325-340 (2001).

15. Mohammed, S.-E.A., Stochastic Functional Differential Equations, Longman Scientific and Technical (1986).

16. Salvadori, L., "Some Contributions to Asymptotic Stability Theory," Ann. Soc. Sci. Bruxellers, Vol. 88, pp. 183-194 (1974).

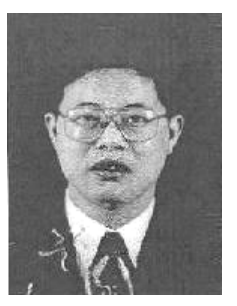

Yi Shen reveived the Ph.D. degree from Huazhong University of Science and Technology, China in 1998 and was then a Post-Doctoral Research Fellow in 1999-2001. He joined the Department of Control Science and Engineering, Huazhong University of Science and Technology, and was made Associated Professor in 2001 which post he still holds. He has presided two National Natural Science Foundation of China (No. 60574025 and 60074008) and over 50 research papers have been published. His main research interests lie in the field of stochastic control and neural network.

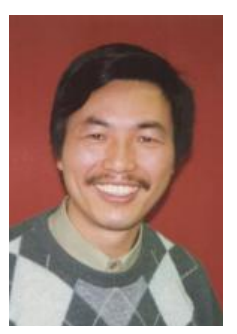

Xuerong Mao reveived the Ph.D. degree from Warwick University, England in 1989 and was then SERC (Science and Engineering Research Council, UK) Post-Doctoral Research Fellow in 19891992. Moving to Scotland, he joined the Department of Statistics and Modelling Science, University of Strathclyde, Glasgow as a lecturer in 1992, was promoted to Reader in 1995, and was made Professor in 1998 which post he still holds. He has authored 3 books and over 150 research papers. His main research interests lie in the field of stochastic analysis. He is a member of the editorial board of Journal of Stochastic Analysis and Applications. 